\title{
Activité protéolytique de souches de lactobacilles thermophiles isolées de levains et de Comté. I. Validation sur minifromages des techniques de laboratoire
}

\author{
Y Bouton*, P Guyot* ${ }^{\star}$ A Dasen, R Grappin
}

\author{
Station de recherches en technologie et analyses laitières, \\ INRA, BP 89, 39801 Poligny Cedex, France; \\ * Comité interprofessionnel du gruyère de Comté, avenue de la Résistance, 39800 Poligny;
}

(Reçu le 16 novembre 1992; accepté le 3 février 1993)

\begin{abstract}
Résumé - Afin d'étudier les relations existant entre les mesures de l'activité protéolytique de souches de bactéries lactiques effectuées en laboratoire et celles observées sur fromages, 6 souches de bactéries lactiques thermophiles isolées de levains et de fromages (Comté) ont été caractérisées selon leur potentiel protéolytique évalué par 2 méthodes : mesure des groupements aminés libres par l'acide 2,4,6 trinitrobenzène sulfonique (TNBS) et détermination de l'activité leucineaminopeptidase (Lap). Des essais en minifabrications de fromages, effectués soit en monosouche soit en associations de souches, ne montrent aucune corrélation significative entre les tests de laboratoire et les mesures de protéolyse réalisées sur fromages avec les méthodes TNBS et Lap.
\end{abstract}

acide 2,4,6 trinitrobenzène sulfonique/ leucine-aminopeptidase/ minifromage/ lactobacille/ protéolyse

Summary - Proteolytic activity of Lactobacilli strains isolated from starters and Comté. I. Validation of laboratory tests on mini-cheeses. Fifty Lactobacillus (helveticus and delbrueckii subsp lactis) strains, isolated from starters and cheeses (Comté), were purified, identified, and classified, according to their acid production level and especially to their proteolytic activity evaluated by two different tests: free amino acids by 2,4,6 trinitrobenzenesulfonic acid (TNBS) and leucineaminopeptidase activity (Lap). Principal component analysis was used for strain grouping. Four Lactobacillus and 2 Streptococcus salivarius subsp thermophilus strains were chosen to study the relationship between the proteolytic activity of lactic bacteria strains assessed with laboratory tests and the nature and extent of proteolysis measured on cheeses. Experimental mini-cheeses were made with single strain and strains associations. Cheeses were made from raw microfiltrated milk to eliminate the influence of original microflora. Sixteen mini-cheeses were made using the conventional Gruyère cheese process. Classification of strains, according to laboratory test results, did not show any significant correlation when the tests were carried out on single or mixed cultures. More data are necessary to obtain a better understanding of the interaction between Streptococci and Lactobacilli. Different classifications of the proteolytic activity of Lactobacilli strains were also obtained when the 
characterization (ie TNBS and Lap methods) was made on mini-cheeses rather than with laboratory tests. Because the origin of the milk, as well as the ripening conditions, may influence the overall cheese proteolysis, model cheeses will have to be developed to study the behaviour of starters.

\section{2,4,6 trinitrobenzenesulfonic acid / leucine-aminopeptidase / mini-cheese / Lactobacillus / pro- teolysis}

\section{INTRODUCTION}

Le Comté est le principal débouché de la production agricole des zones de plateaux et de montagne de Franche-Comté. Avec une production annuelle de $38000 \mathrm{t}$ réalisée par près de 250 ateliers de fabrication (ou fruitières), il se place au premier rang des fromages d'appellation d'origine contrôlée. Ces fruitières travaillent de façon traditionnelle, utilisant notamment des ferments lactiques non sélectionnés, dits "sauvages", avec apport occasionnel de ferments commerciaux. La grande variabilité de la qualité de la matière première et des techniques de fabrication, associée au manque de maîtrise des ferments lactiques, a des répercussions directes sur la qualité et la régularité du produit. Par ailleurs, dans le contexte économique actuel, le Comté doit prouver sa spécificité aussi bien à travers ses qualités organoleptiques que par son attachement au terroir, justifiant son appellation d'origine contrôlée. C'est dans ce contexte que la profession fromagère régionale a décidé de créer une collection de souches locales de bactéries lactiques qui soient sélectionnées d'après leurs aptitudes technologiques et organoleptiques, pour être utilisées régulièrement en fabrication de Comté.

On sait que la protéolyse est le mécanisme le plus important de la plupart des fromages, car il affecte à la fois la texture et la flaveur (Fox, 1989). Or les levains lactiques, s'ils abaissent le $\mathrm{pH}$ du milieu en transformant le lactose en acide lactique, contribuent également aux caractères organoleptiques des fromages en libérant des systèmes enzymatiques, en particulier protéolytiques, qui participent aux principaux phénomènes de l'affinage (Thomas et Pritchard, 1987). En complétant l'action de la présure et celle de la plasmine, les enzymes protéolytiques des bactéries lactiques jouent un rôle fondamental dans les fromages; cet équipement protéolytique important est actuellement bien décrit (Desmazeaud, 1990). Deux catégories d'enzymes peuvent être répertoriées : les protéinases qui scindent les protéines en gros et moyens peptides, et les peptidases qui dégradent les peptides issus de la dégradation des protéines en acides aminés. Ainsi, pour caractériser un système enzymatique aussi complexe, on peut avoir recours soit à des techniques qui mesurent le degré de protéolyse en identifiant les protéines hydrolysées et les produits d'hydrolyse, soit en mesurant une activité protéolytique spécifique de type exopeptidase ou endopeptidase, sur substrats synthétiques ou sur différentes caséines.

L'activité protéolytique globale, liée à la formation de groupements $-\mathrm{NH}_{2}$ libres, a souvent été mesurée, soit à l'aide du réactif à la ninhydrine, soit en utilisant des réactifs tels que l'ortho-phthaldialdehyde ou oPA (Oberg et al, 1991), I'acide 2,4,6 trinitrobenzènesulfonique ou TNBS (Mc Kellar, 1981; Rollema et al, 1989). La chromatographie liquide haute performance a également été utilisée pour caractériser l'activité protéolytique de souches (Oberg et al, 1990). Pour mesurer de manière spécifi- 
que une activité protéolytique particulière, les substrats synthétiques choisis ont été couplés à des groupements paranitroanilide ou $\beta$-naphtylamide (El Soda et Desmazeaud, 1982; Cholette et Mc Kellar, 1990). Si ces techniques permettent de mieux appréhender le potentiel protéolytique des souches cultivées sur lait ou milieu synthétique, qu'en est-il dans le fromage ? Peut-on, en effet, prévoir à partir des résultats observés au laboratoire si les bactéries ont toujours la même activité sur fromage?

Au cours de ce travail, nous avons donc tenté, d'une part, d'établir les liens existant entre l'activité protéolytique de souches et associations de souches de bactéries lactiques évaluée sur lait et milieu synthétique et le niveau de protéolyse des fromages fabriqués à partir de ces mêmes souches et, d'autre part, de préciser l'influence des levains sur les caractéristiques physicochimiques et sensorielles du Comté. Pour mesurer la protéolyse, deux techniques ont été choisies en raison de leur simplicité de mise en œuvre sur cultures de souches et sur fromages : le dosage des groupements aminés $\left(-\mathrm{NH}_{2}\right)$ qui est une mesure globale de la protéolyse, par l'acide 2,4,6 trinitrobenzènesulfonique (TNBS), et une mesure d'activité peptidasique largement répandue chez les bactéries lactiques (Bouillanne et Desmazeaud, 1980; Meyer et al, 1989 ; Khalid et Marth, 1990), l'activité leucine-aminopeptidasique (Lap) sur substrat spécifique (leucine-paranitroanilide).

Dans ce premier article, après une présentation des techniques utilisées pour évaluer le potentiel protéolytique des souches, nous comparerons les résultats obtenus sur milieu lait ou synthétique aux résultats obtenus sur minifromages. L'influence des levains sur les caractéristiques physico-chimiques et sensorielles du Comté sera donnée dans un second article.

\section{MATÉRIEL ET MÉTHODES}

\section{Souches de bactéries lactiques}

Après avoir choisi des ateliers utilisant exclusivement des levains thermophiles sauvages et produisant des fromages de haute qualité organoleptique, de bonne présentation et ayant une grande aptitude à la conservation, le premier travail a consisté à isoler, purifier et identifier les souches de bactéries lactiques à partir de levains et de Comté; puis, dans un second temps, à les caractériser selon leur aptitude à l'acidification et surtout leur pouvoir protéolytique. Les bactéries utilisées dans cette étude sont issues de cette collection ; il s'agit de 45 souches de $L$ helveticus, 5 souches de $L$ delbrueckii subsp lactis et 2 souches de $S$ salivarius subsp thermophilus (S69 et S48). . Nous disposons sur ces souches, en plus des mesures d'activité protéolytique présentées dans cet article, d'un ensemble d'informations portant sur leur potentiel acidifiant (à $44^{\circ} \mathrm{C}$ et en cycle thermique), et sur leur profil biochimique déterminé à l'aide des galeries API $50 \mathrm{CH}$ (Bouton, 1992). Les souches sont conservées à $-80^{\circ} \mathrm{C}$ dans un milieu synthétique M17 ou MRS (selon qu'il s'agisse de streptocoques ou de lactobacilles), additionné de glycérol stérile à la concentration de $15 \%$ (v/v). Avant chaque test, les souches ont été remises en culture et repiquées 2 à 3 fois sur lait écrémé stérile spray instantané, exempt d'antibiotique (ULN, Neuilly-sur-Seine, France), reconstitué à $10 \%$ (Bouillanne et Desmazeaud, 1980).

L'évaluation du potentiel protéolytique a été réalisée sur des monosouches (culture d'une seule souche de bactérie) et sur des associations de souches (cultures mixtes, composées d'une souche de lactobacilles et d'une ou deux souches de streptocoques), afin d'assurer une meilleure transformation du lactose en acide lactique et observer une éventuelle synergie sur l'activité protéolytique.

\section{Fabrications fromagères}

Les fabrications fromagères ont été réalisées dans un atelier comprenant 4 minicuves. Chaque jour de fabrication, 4 fromages ont été fabriqués à partir du même lait, chaque cuve étant 
ensemencée avec une souche ou une association de souches différente. Mis à part le facteur souche qui variait entre fromages, l'ensemble des opérations de fabrication et d'affinage était identique. La comparaison physico-chimique des fromages (extrait sec, teneur en $\mathrm{NaCl}$ et taux de matière grasse) fabriqués le même jour. très homogène ( $\mathrm{CV}<1 \%$ ), indique que le processus de fabrication des fromages (coagulation, décaillage, synérèse et pressage) est bien maîtrisé. Chaque souche a fait l'objet de 2 essais effectués avec des laits de traites différentes dont la composition physico-chimique et la qualité microbiologique pouvaient être légèrement diffèrentes. Afin d'éliminer en grande partie l'influence éventuelle de la flore originelle du lait, et pour se placer dans des conditions de fabrication du Comté au lait cru, les essais en atelier expérimental ont été réalisés sur des laits microfiltrés dont le niveau de flore était en moyenne de $10^{3} \mathrm{UFC} / \mathrm{ml}$.

Le lait, stocké 12 ou $24 \mathrm{~h}$ à $+4^{\circ} \mathrm{C}$, est collecté dans une fromagerie proche de l'atelier expérimental. À son arrivée, il est écrémé puis microfiltrè à l'aide d'un appareil Bactocatch (AlfaLaval, Les Clayes-sous-Bois, France) d'un débit de $600 \mathrm{l} / \mathrm{h} / \mathrm{m}^{2}$. L'élimination des bactéries est réalisée à ufroid" $\left(35^{\circ} \mathrm{C}\right)$ par microfiltration tangentielle, la taille des pores et la surface de la membrane étant respectivement de 1,4 $\mu \mathrm{m}$ et $0,2 \mathrm{~m}^{2}$. Les rendements de microfiltration généralement observés sont voisins de $99,5 \%$. La crème recueillie est pasteurisée $\left(85^{\circ} \mathrm{C}, 30 \mathrm{~s}\right)$ et réincorporée au lait microfiltré (standardisation en matière grasse à 30-31 g/l). Les fromages sont fabriqués à partir de $11 \mathrm{~kg}$ de lait. L'ensemencement en levains thermophiles (addition de $0,12 \%$ de streptocoques et $0,08 \%$ de lactobacilles en association de souches et $0,10 \%$ en monosouche) est réalisé pendant la maturation à $32,5^{\circ} \mathrm{C}, 30 \mathrm{~min}$. L'emprésurage à $32,5^{\circ} \mathrm{C}$ est effectué avec de la présure à $700 \mathrm{mg}$ de chymosine/l (Granday, Beaune, France) ; la quantité d'enzyme à ajouter est déterminée par le test de Berridge, afin d'obtenir un temps de prise voisin de $30 \mathrm{~min}$. La coagulation est mesurée à l'aide d'une sonde thermique (Coagulometre INRA, Brevet Fr 88-00803).

Le décaillage commence 5 min après la coagulation et dure $4 \mathrm{~min}$. La température s'élève ensuite jusqu'à $54^{\circ} \mathrm{C}$ en $35 \mathrm{~min}$ et reste constante pendant $25 \mathrm{~min}$. Le soutirage se fait par gravité et le pressage $\left(150 \mathrm{~g} / \mathrm{cm}^{2}\right.$, pendant $6 \mathrm{~h}$ ) a lieu dans une enceinte thermostatée qui si- mule le cycle thermique de refroidissement réel rencontré en industrie sur gros fromages. Afin d'obtenir des conditions d'acidification normales, la température s'abaisse lentement (en $16 \mathrm{~h}$ ) avant de se stabiliser à $31^{\circ} \mathrm{C}$ pendant $4 \mathrm{~h}$. Le salage est effectué à $13^{\circ} \mathrm{C}$ pendant $3 \mathrm{~h}$, dans une saumure saturée en $\mathrm{NaCl}, d=1190, \mathrm{pH}$ 5,20 . Les minifromages (diamètre $15 \mathrm{~cm}$, masse $950 \mathrm{~g})$ sont ensuite recouverts de paraffine. L'affinage est conduit dans 2 cellules dont la température et l'hygrométrie sont régulées automatiquement (Cardenas et al, 1991a et b) ; tout d'abord en cave de préaffinage, à $14^{\circ} \mathrm{C}$ pendant 3 semaines, où thumidité relative est de 92 à $94 \%$, puis en cave chaude à $18^{\circ} \mathrm{C}$ pendant 9 semaines (humidité relative $>94 \%$ ).

\section{Dosage de l'activité protéolytique}

\section{Dosage des groupements aminés à l'aide du TNBS}

\section{Sur cultures de souches de bactéries lactiques}

Le dosage des radicaux $-\mathrm{NH}_{2}$ libres est réalisé selon une technique adaptée de la méthode de Mc Kellar (1981). Vingt $\mathrm{ml}$ de lait $\mathrm{G}$ (Prolait, Niort, France) reconstitué à $10 \%$, ensemencés à $1 \%(\mathrm{v} / \mathrm{v})$ soit environ $210^{6} \mathrm{UFC} / \mathrm{ml}$, sont incubés à $44^{\circ} \mathrm{C}$. Des prélèvements de $2 \mathrm{ml}$ sont effectués au bout de $0,2,4,6,8$ et $24 \mathrm{~h}$ d'incubation. A chaque prélèvement sont ajoutés $4 \mathrm{ml}$ de TCA $0,72 \mathrm{~N}$. Après $20 \mathrm{~min}$ à température ambiante, le précipité est séparé du surnageant par centrifugation à $1200 \mathrm{~g}$ pendant $10 \mathrm{~min}$ à $5^{\circ} \mathrm{C}$, puis filtré sur papier whatman $n^{\circ} 42$. $\AA$ $100 \mu$ de filtrat sont ajoutés $1 \mathrm{ml}$ de tampon borate de sodium $0,1 \mathrm{~mol} / \mathrm{I}, \mathrm{pH} 9,5$ et $400 \mu \mathrm{l}$ de solution de TNBS à $100 \mu \mathrm{g} / \mathrm{ml}$ (Sigma P2297). Après $1 \mathrm{~h}$ d'incubation à $37^{\circ} \mathrm{C}$ à l'abri de la lumière, la réaction est stoppée par addition de $0,4 \mathrm{ml}$ d'une solution de phosphate/sulfite de sodium (respectivement 2 et $18 \mathrm{mmol} / \mathrm{l})$, préparée extemporanément. L'absorbance est mesurée à $420 \mathrm{~nm}$ après $15 \mathrm{~min}$ par référence à un blanc réalisé avec de l'eau distillée; les résultats sont exprimés en mmol d'équivalent glycine par litre de lait (meq gly/l) à l'aide d'une gamme étalon de solutions de glycine de 0,01 à $0,5 \mathrm{mmol} / \mathrm{l}$.

Les ensemencements à $1 \%(\mathrm{v} / \mathrm{V})$ ont été réalisés dans le cas de monosouche à partir d'une 
seule culture mère, et dans le cas des associations de souches à partir des différentes cultures mères composant cette association.

\section{Sur fromages}

Les échantillons de fromages prélevés après 12 semaines d'affinage ont été emballés dans du papier d'aluminium sulfurisé (l'aluminium étant du côté du fromage, afin de limiter les transferts de soluté lors de la congélation), puis congelés à $-25^{\circ} \mathrm{C}$. Le dosage est effectué sur des dilutions au $1 / 100$ de préparation d'azote soluble dans l'eau réalisée selon une technique adaptée de la méthode de Kuchroo et Fox (1982). Les résultats sont exprimés en équivalent glycine, converti en $\mathrm{g}$ d'azote, pour $100 \mathrm{~g}$ d'azote total $\left(-\mathrm{NH}_{2} / \mathrm{NT}\right)$. L'azote total est déterminé par la méthode Kjeldahl.

\section{Détermination de l'activité leucine-aminopeptidasique}

\section{Sur cultures de souches de bactéries lactiques}

La mesure de cette activité est réalisée selon la technique de Rabier et Desmazeaud (1973) à partir du substrat : L-leucine-paranitroanilide. Quatre-vingt $\mathrm{ml}$ de milieu synthétique $\mathrm{M} 17$ ou MRS, selon le cas, sont ensemencés à $2 \%$ ( $\mathrm{V} /$ v), puis incubés à $44^{\circ} \mathrm{C}$ pendant $6 \mathrm{~h}$ pour les streptocoques ou $8 \mathrm{~h}$ pour les lactobacilles : dans le cas des associations de souches, l'ensemencement à $2 \%$ est réalisé sur milieu Elliker, à partir des différentes cultures mères composant cette association; l'incubation à $44^{\circ} \mathrm{C}$ dure $8 \mathrm{~h}$. La culture est ensuite centrifugée à $1200 \mathrm{~g}$ pendant $20 \mathrm{~min}$ à $5^{\circ} \mathrm{C}$; le culot cellulaire est lavé 2 fois à l'aide d'une solution d'eau physiologique stérile. Le dosage est réalisé sur des suspensions de cellules, ajustées à une absorbance de 3 , à $650 \mathrm{~nm}$ correspondant à une concentration d'environ $210^{8}$ bactéries $/ \mathrm{ml}$ (la concentration cellulaire est déterminée par turbidimétrie, à l'aide d'un spectrophotomètre Beckman DU 64). Pour chaque souche, 4 tubes contenant le milieu réactionnel suivant sont préparés : $100 \mu \mathrm{l}$ de suspension cellulaire (absorbance $=3$ ); $900 \mu \mathrm{l}$ de tampon phosphate de sodium $0,05 \mathrm{~mol} / \mathrm{l}, \mathrm{pH}=7$ et $50 \mu \mathrm{l}$ de substrat $(6,5$ $\mathrm{mg}$ de L-leucine-p-nitroanilide, Sigma L9125, dans $1 \mathrm{ml}$ de méthanol pur).
Après incubation à $37^{\circ} \mathrm{C}$ pendant 10,20 et $30 \mathrm{~min}$, la réaction est stoppée par addition de $250 \mu \mathrm{l}$ d'acide acétique à $30 \%(\mathrm{v} / \mathrm{v})$. Après centrifugation à $1200 \mathrm{~g}$ pendant $5 \mathrm{~min}$ à $5^{\circ} \mathrm{C}$, la variation d'absorbance est mesuree à $410 \mathrm{~nm}$ par référence à un tube témoin, dans lequel la suspension cellulaire a été remplacée par de l'eau physiologique. Les résultats d'activité aminopeptidasique sont exprimés en $\mu \mathrm{g}$ de $p$-nitroaniline libéré par heure, à l'aide d'une gamme étalon.

\section{Sur fromages}

Le substrat (leucine-p-nitroanilide) est dissous dans du diméthylformamide, puis dilué dans du tampon tris/acide maléique/soude $(50 \mathrm{mmol} /$, $\mathrm{pH} 5,5)$, de façon à obtenir une concentration de $510^{-5} \mathrm{~mol} / \mathrm{l}$ en substrat et de $10 \%$ en diméthylformamide (Linden et al, 1982). A $0,5 \mathrm{ml}$ de broyat de fromage, réalisé dans de l'eau citratée à $2 \%$, sont ajoutés $1,5 \mathrm{ml}$ de la solution de substrat. Après incubation, la lecture est réalisée au spectrophotomètre à $410 \mathrm{~nm}$, après dissolution totale du milieu réactionnel par addition de transparisant (Prolabo, ref 27 357.232). Les résultats sont exprimés en $\mu \mathrm{g}$ de p-nitroaniline libéré par $\mathrm{g}$ de fromage et par heure.

\section{Fidélité des mesures}

Elle constitue un élèment indispensable à connaitre avant d'entreprendre tout travail analytique. L'étude de la répétabilité et de la reproductibilité permet d'affecter à chaque valeur une incertitude de mesure. La validité des résultats peut ainsi être vérifiée lorsque les mesures sont par exemple réalisées en double. Les calculs ont été effectués selon la norme FIL (Anonyme, 1985).

\section{Technique TNBS}

La répétabilité des mesures, ou plus exactement de la réaction chimique, a étè évaluée à partir de filtrats analysés en double le même jour, provenant de 7 souches différentes. Pour évaluer la reproductibilité dans le temps, un dosage des groupements aminés a été réalisé en simple sur 3 séries de filtrats obtenus pendant 3 jours différents, à partir des cultures en monosouche (7 échantillons). 
Aux écarts types de répétabilité (tableau I), voisins de 0,01 meq gly/l de lait, sauf au temps T24 où la valeur est de 0,063 meq gly/l de lait, correspondent des $\mathrm{CV}$ de $2 \%$, pour des moyennes $\geq 0,500$ meq gly/l de lait. Les CV de reproductibilité, qui incluent l'étape d'inoculation et d'incubation, sont de 4 à 10 fois plus élevés que les $\mathrm{CV}$ de répétabilité. La reproductibilité est meilleure à T24, temps pour lequel le maximum de protéolyse est observé ; c'est ce temps qui sera retenu pour comparer les souches entre elles.

\section{Technique Lap}

La répétabilité des mesures, et plus précisément de la réaction enzymatique, a été déterminée à partir de 8 préparations issues de 8 souches différentes analysées en double le même jour. La reproductibilité a été estimée à partir de 3 déterminations de l'activité aminopeptidasique, effectuées en simple pendant 3 jours différents sur 10 monosouches ( 3 streptocoques et 7 lactobacilles).

En répétabilité (tableau I), le coefficient de variation obtenu est similaire à celui observé par Linden et al (1982) sur le lait $(\mathrm{N}=32, \mathrm{CVr}=$ $4,97 \%)$. En reproductibilité, le coefficient de variation de $14,6 \%$ est du même ordre de grandeur que celui obtenu pour la mesure des groupements aminés par la technique du TNBS après une incubation de $8 \mathrm{~h}$.

\section{Traitements des résultats}

Les analyses statistiques ont été effectuées à partir du logiciel Stat-ITCF (Version 4, 1987) de I'Institut technique des céréales et des fourrages.

Pour classer les souches bactériennes selon leur pouvoir protéolytique, nous avons employé une méthode d'analyse multidimensionnelle, l'analyse en composantes principales ou ACP (Zourari et al, 1991). La comparaison de la protéolyse observée sur souches et sur minifromages a été réalisée par analyse de variance. Lorsque le facteur souche s'est révélé significatif, le test de Newman-Keuls a été appliqué afin de classer les souches entre elles; ce classement est basé sur la plus petite différence existant entre les différentes moyennes.

Tableau I. Répétabilité et reproductibilité dans le temps des techniques TNBS et Lap.

Repeatability and between-day reproducibility of TNBS and Lap technics.

\begin{tabular}{|c|c|c|c|c|c|c|c|c|c|c|c|c|}
\hline & \multicolumn{5}{|c|}{ Répétabilité } & \multicolumn{7}{|c|}{ Reproductibilité } \\
\hline & \multicolumn{5}{|c|}{$\operatorname{TNBS}(\mathbb{N}=7)$} & \multirow{2}{*}{$\begin{array}{c}\text { Lap } \\
(\mathrm{N}=8)\end{array}$} & \multicolumn{4}{|c|}{$\operatorname{TNBS}(\mathrm{N}=7)$} & \multicolumn{2}{|c|}{$\begin{array}{c}\operatorname{Lap} \\
-(N=10)\end{array}$} \\
\hline & $T 2$ & $T 4$ & $T 6$ & T8 & T24 & & $T 2$ & T4 & T6 & T8 & T24 & \\
\hline $\bar{x}$ & 0,103 & 0,193 & 0,531 & 0,859 & 3,359 & 21,86 & 0,07 & 0,16 & 0,41 & 0,90 & 3,29 & 22,86 \\
\hline s & 0,007 & 0,007 & 0,011 & 0,010 & 0,063 & 0,88 & 0,02 & 0,04 & 0,08 & 0,14 & 0,23 & 3,33 \\
\hline CV & 6,6 & 3,6 & 2,0 & 1,9 & 1,9 & 4,0 & 29,2 & 26,5 & 20,2 & 15,7 & 6,9 & 14,6 \\
\hline
\end{tabular}

$\bar{x}$ : moyenne et $s:$ écart type (TNBS : meq gly/l de lait; Lap : $\mu \mathrm{g}$ p-nitroaniline/h); CV : coefficient de variation en \%; N : nombre d'échantillons; T2, T4, T6, T8, T24 :mesures TNBS respectivement au bout de $2,4,6,8$ et $24 \mathrm{~h}$ d'incubation.

$\bar{x}$ : mean and $s$ : repeatability standard deviation (TNBS : meq gly/l milk; Lap : $\mu g$ p-nitroaniline/h); $\mathrm{CV}$ : coefficient of variation in \%; $N$ : number of samples; T2, T4, T6, T8, T24: TNBS measurements respectively after $2,4,6,8$ and 24 h of incubation. 


\section{RÉSULTATS ET DISCUSSION}

\section{Caractérisation et choix des souches}

L'ACP, réalisée à partir des mesures TNBS et Lap, a permis de classer les 50 souches de lactobacilles sur la base de leur potentiel protéolytique, sachant qu'il n'existe pas de corrélation entre les 2 techniques $(r=0,04)$. Les axes 1 et 2 expliquent $100 \%$ de la variance ; la projection du nuage de points sur un plan est présentée sur la figure 1. Les différentes souches sont réparties selon 2 axes, décrits par les variables Lap et TNBS; les souches 116, $111,57,75$ et 72 sont celles qui présentent l'activité Lap la plus élevée (35,9 à 40 $\mu \mathrm{g}$ de $\mathrm{p}$-nitroaniline/h); à l'opposé, les souches $131,85,9,139$ et 90 sont celles qui ont des valeurs de Lap faibles (5,5 à $12,7 \mu \mathrm{g}$ p-nitroaniline/h).

De la même manière, des groupes de souches peuvent être constitués à partir des résultats TNBS, même si la distinction entre souches moyennes et souches faibles ou souches moyennes et souches fortes n'est pas toujours aisée. II ressort que les souches $16,42,40$ et 27 sont celles qui présentent avec les souches 90 , 72 et 75 les valeurs les plus hautes $(3,65$ à 5,18 meq gly/l de lait), tandis qu'à l'opposé se situent les souches $85,131,123,99$, 137 et 113 présentant les valeurs les plus faibles ( 0,86 à 1,74 meq gly/l de lait).

Afin de réaliser des fabrications fromagères simultanément sur les souches sélectionnées, il a été décidé de retenir 4 souches de lactobacilles, présentant des

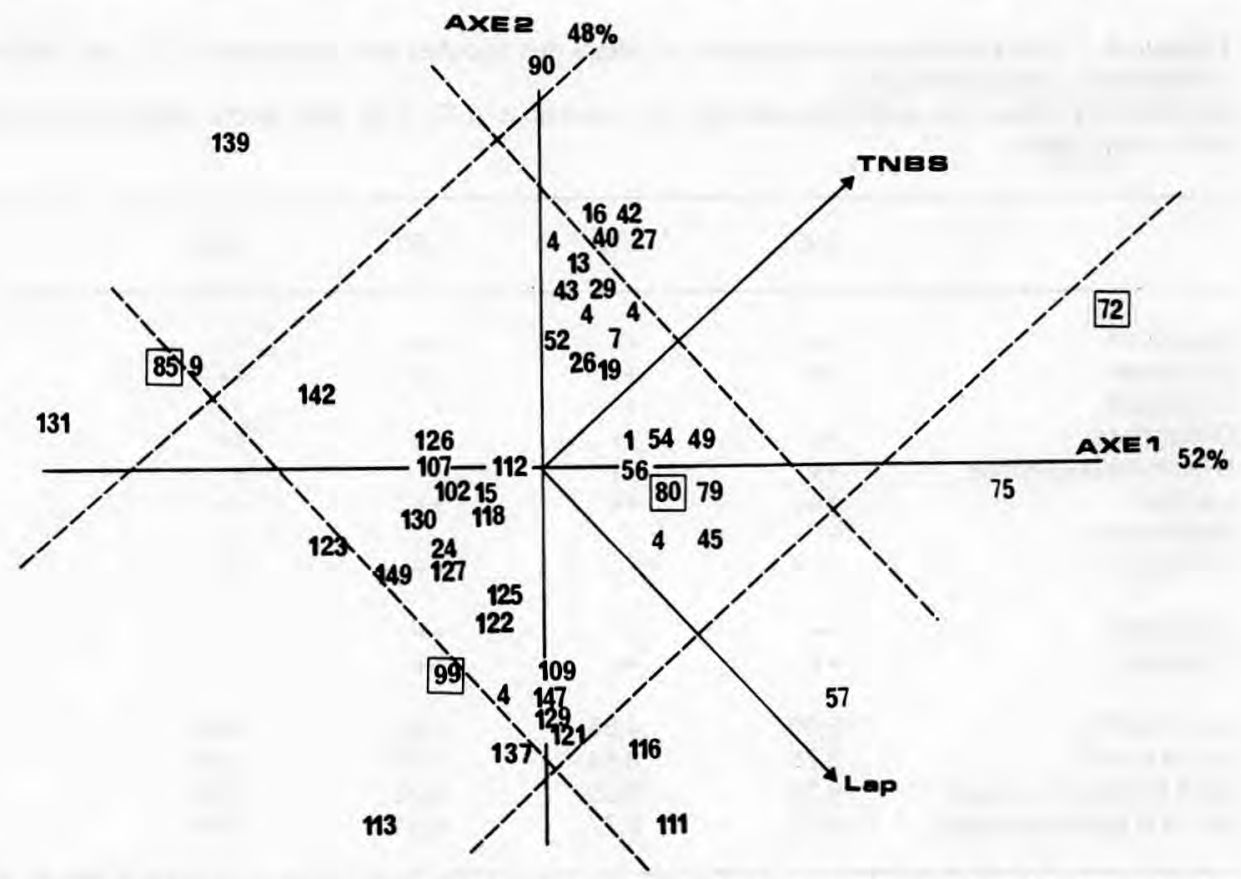

Fig 1. Analyse en composantes principales pour les souches de Lactobacillus. TNBS : détermination des groupements aminés par la méthode TNBS; Lap : activité leucine-aminopeptidasique.

Principal component analysis for Lactobacillus strains; TNBS : amino group determination by TNBS method; Lap : leucine-aminopeptidase activity. 
valeurs d'activité protéolytique extrêmes : $L$ helveticus $L 72$ qui présente des valeurs élevées aux 2 activités, $L$ delbrueckii subsp lactis $\mathrm{L} 85$ qui présente au contraire de faibles valeurs, $L$ helveticus $L 80$ qui a donné des valeurs moyennes et $L$ helveticus L99 qui a donné une faible valeur au dosage TNBS et un résultat moyen pour l'activité Lap. Nous avons vérifié que l'acidification de ces souches était correcte $(\mathrm{pH}$ voisin de 4,5 après $8 \mathrm{~h}$ d'incubation à $44^{\circ} \mathrm{C}$ ) et qu'elles présentaient également des profils biochimiques différents, afin d'éviter de prendre des souches identiques qui peuvent provenir de la même bactérie mère (tableau II). Soulignons qu'il n'existe aucune relation entre l'origine de ces souches (fromagerie, levain, fromage) et leurs caractéristiques protéolytiques.
Les 4 souches de lactobacilles ont été associées soit séparément, soit ensemble, à 2 souches de $S$ salivarius subsp thermophilus ( $\mathbf{S} 69$ et $\mathrm{S} 48$ ) constituant ainsi 12 associations différentes. Les souches de streptocoques utilisées présentent des valeurs moyennes d'activité Lap (respectivement 14,7 et $13,7 \mu \mathrm{g}$ p-nitroaniline/h). Les résultats des dosages TNBS furent semblables à ceux du témoin, suggérant que la protéolyse produite par les streptocoques est négligeable ou en tout cas trop limitée pour être mesurée par cette technique (tableau III). Ces résultats sont en accord avec les observations faites par Gripon (1986). La souche S69 acidifie fortement au bout de $6 \mathrm{~h}$ de culture par rapport à la souche $\mathrm{S} 48$; ces 2 souches présentent un profil biochimique différent (tableau III).

Tableau II. Profils biochimiques et potentiel acidifiant des souches de $L$ helveticus $(L 72, L 80, L 99)$ et L delbrueckii subsp lactis (L85).

Biochimical profiles and acidifying potential of $L$ helveticus $(L 72, L 80, L 99)$ and $L$ delbrueckii subsp lactis (L85) strains.

\begin{tabular}{|c|c|c|c|c|}
\hline & L99 & $L 72$ & L80 & $L 85$ \\
\hline Galactose & ++ & ++ & ++ & + \\
\hline D-glucose & ++ & ++ & ++ & ++ \\
\hline D-fructose & - & + & + & ++ \\
\hline D-mannose & ++ & ++ & ++ & ++ \\
\hline $\mathrm{N}$-acétyl-glucosamine & ++ & ++ & + & + \\
\hline Lactose & ++ & ++ & ++ & ++ \\
\hline Saccharose & - & - & - & ++ \\
\hline Tréhalose & - & - & ++ & ++ \\
\hline D-lactates & ++ & ++ & ++ & ++ \\
\hline L-lactates & ++ & ++ & ++ & - \\
\hline $\mathrm{pH} 8 \mathrm{~h} 44^{\circ} \mathrm{C}$ & 5,00 & 4,35 & 4,62 & 4,64 \\
\hline $\mathrm{pH} 24 \mathrm{~h} 44^{\circ} \mathrm{C}$ & 3,75 & 3,64 & 3,73 & 3,98 \\
\hline $\mathrm{pH} 8 \mathrm{~h}$ cycle thermique & 6,39 & 6,25 & 6,23 & 6,48 \\
\hline $\mathrm{pH} 24 \mathrm{~h}$ cycle thermique & 4,03 & 5,77 & 4,51 & 4,39 \\
\hline
\end{tabular}

Tous les autres composés des galeries $50 \mathrm{CH}$ (Api-Biomérieux) étaient négatifs; (-) test négatif (++) test positif, (+) test faiblement positif. Les mesures de pH ont été obtenues après 8 et $24 \mathrm{~h}$ d'incubation dans le lait à $44^{\circ} \mathrm{C}$ ou après cycle thermique.

All the other $50 \mathrm{CH}$ tests were negative; $(-)$ negative test; $(++)$ positive test, $(+)$ weakly positive test. pH measurements are obtained after 8 and $24 \mathrm{~h}$ incubation in milk at $44^{\circ} \mathrm{C}$ or after thermal cycle. 
Tableau III. Profils biochimiques, potentiel acidifiant et protéolytique des souches de Streptococcus salivarius subsp thermophilus (S69, S48).

Biochimical profiles, aciditying and proteolytic potential of Streptococcus salivarius subsp thermophilus (S69, S48) strains.

\section{$\$ 69 \quad 548$}

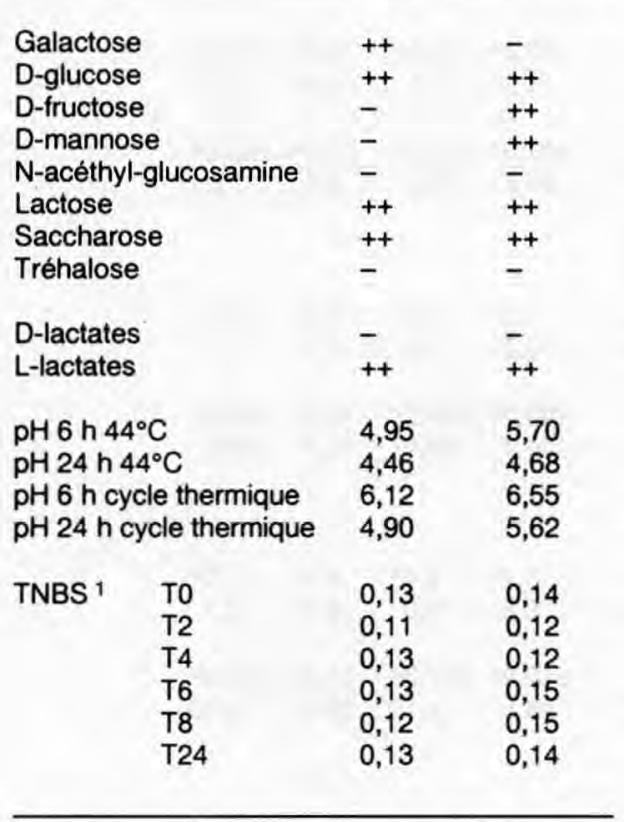

Tous les autres composés des galeries $50 \mathrm{CH}$ (ApiBiomérieux) étaient négatifs; $(-)$ test négatif, $(++)$ test positif, (+) test faiblement positif. (1) mesures TNBS en absorbance; l'absorbance du témoin (lait non ensemencé) est de 0,13 . Les mesures de $\mathrm{pH}$ ont été obtenues après 8 et $24 \mathrm{~h}$ d'incubation dans le lait à $44^{\circ} \mathrm{C}$ ou après cycle thermique.

All the other $50 \mathrm{CH}$ tests were negative; $(-)$ negative test, $(++)$ positive test, (+) weakly positive test. (1) TNBS measurements in absorbance; the reference optical density (uninoculated milk) is 0.13 . $\mathrm{pH}$ measurements are obtained after 8 and $24 \mathrm{~h}$ incubation in milk at $44^{\circ} \mathrm{C}$ or after thermal cycle.

\section{Activité protéolytique des souches et associations de souches}

Nous considérerons les dosages TNBS et l'activité Lap effectués sur monosouches et associations de souches, d'une part, sur cultures au laboratoire et, d'autre part, sur fromages à 3 mois pour le TNBS et à $20 \mathrm{~h}$ pour l'activité Lap. À partir de ces données, une étude du facteur "lactobacille" a été réalisée à l'aide d'une analyse de variance (tableau IV) selon un plan factoriel comportant 4 niveaux (souches)' et respectivement 3 répétitions pour les tests de laboratoire et 2 répétitions pour les tests sur fromages.

\section{Comparaison de la protéolyse observée au laboratoire sur cultures de souches et associations de souches}

D'une façon générale, l'activité protéolytique globale (valeurs TNBS) obtenue en association de souches est beaucoup plus faible et plus homogène qu'en monosouche. Le dosage sur cultures de monosouche permet un classement en 3 groupes : L72 (groupe a) > L80 (groupe b) $>$ L85 et L99 (groupe c) ; en associations, la souche $\mathrm{L} 72$ est toujours la première (groupe a), les autres souches constituant un autre groupe homogène (groupe b) ; la souche $\mathrm{L} 80$ se retrouve ainsi en association de souches, au même niveau que les souches L85 ou L99, alors qu'elle exprimait une protéolyse plus importante en monosouche. Dans le dernier cas (mélanges avec S69 et S48), les écarts types de répétabilité calculés pour chacune des associations n'étant pas identiques, il est impossible de conclure. Toutefois, la hiérarchie obtenue lors des 3 répétitions a toujours été la même, le mélange présentant la souche $\mathrm{L} 72$ restant le plus protéolytique. On voit donc que si les associations contenant la souche L72 (souche forte- 
Tableau IV. Analyse de variance des mesures de protéolyse (TNBS et Lap) effectuées sur souches et minifromages de 3 mois.

Analysis of variance of proteolysis measurements (TNBS and Lap) assessed on strains and minicheeses aged 3 months.

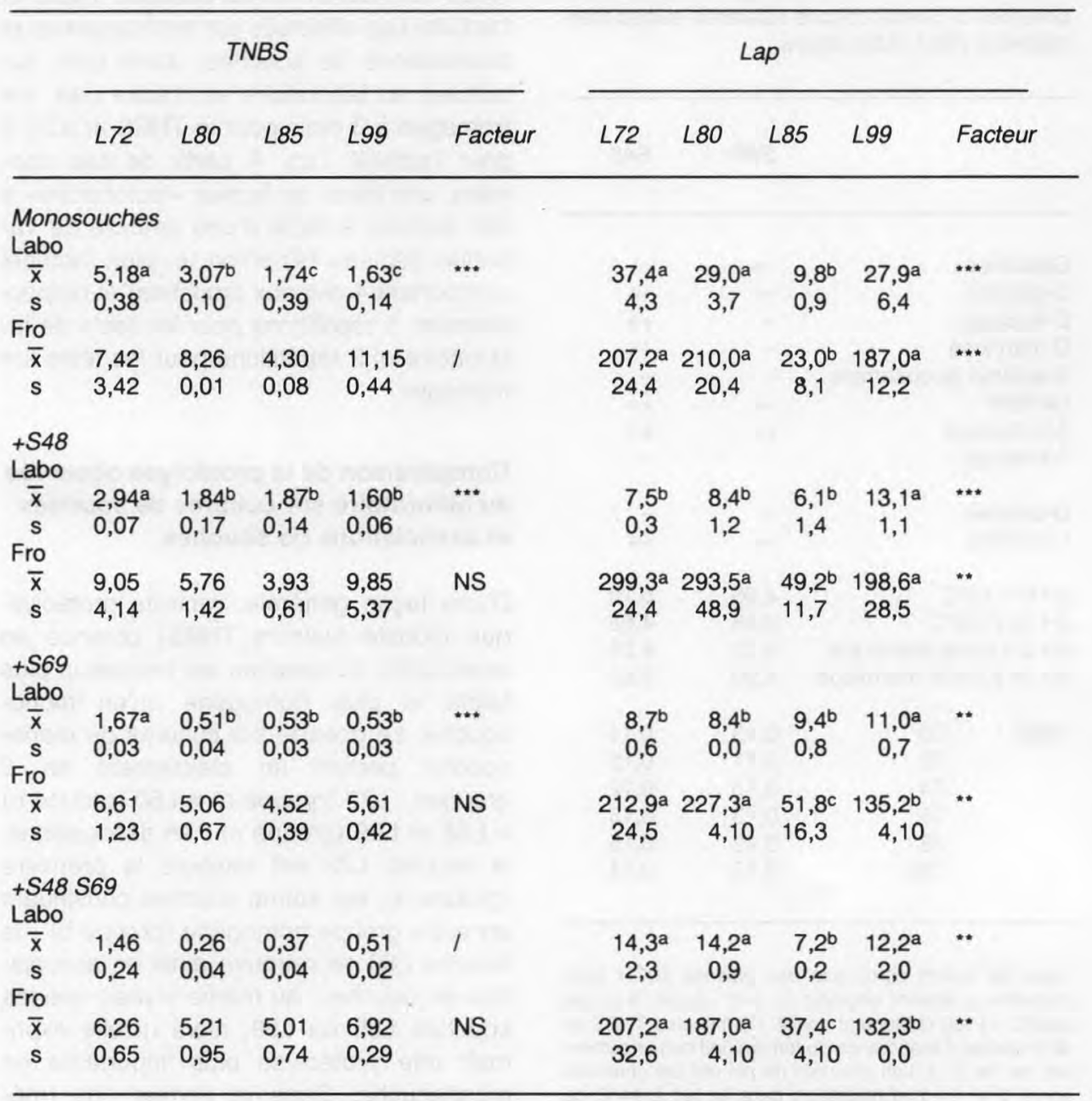

$\overline{\mathrm{x}}$ : moyenne; s : écart type; seuil de signification du facteur souche, non significatif (NS), significatif au seuil de $1 \%$ $(* *)$, de $0,1 \%\left({ }^{* * *}\right)$; le test de Newman-Keuls conduit à un classement (décroissant) des souches en différents groupes homogènes symbolisés par les lettres a, b, c: (/) aucune conclusion n'a pu être donnée, les variances entre traitements n'étant pas identiques.

$\bar{x}$ : mean; s : standard deviation; significant level of factor strain, non significant (NS), significant $P<0.01$ (**), significant $P<0.001\left({ }^{* * *}\right) ;$ Newman-Keuls'test leads to strains classification (decreasing proteolytic intensity) in different groups noted as $a, b, c ;()$ the variance between treatments being unhomogeneous, no conclusion can be drawn. 
ment protéolytique) sont bien celles qui donnent une activité protéolytique globale plus élevée, la distinction n'est pas franche avec les mélanges dans lesquels les souches L80, L85 et L99 sont présentes. En comparant les associations constituées uniquement de 2 souches, il ressort que les associations dans lesquelles la souche S48 est présente sont celles qui donnent une activité protéolytique globale plus forte.

Avec la technique Lap, on observe le même type d'information en association de souches, à savoir des résultats plus faibles et un nivellement des valeurs. La souche L72 par exemple, plus protéolytique que la souche L80 en monosouche, ne se distingue plus guère des autres souches de lactobacilles lorsqu'elle est associée à des streptocoques. Toutefois, avec la souche L85, contrairement aux autres souches de Lactobacillus, les variations des valeurs Lap observées entre monosouche et association sont moins importantes. Les résultats des mesures Lap montrent que les mélanges formés avec cette souche L85 donnent généralement les valeurs les plus faibles (sauf dans le cas du mélange L85 S69); en revanche, les associations dans lesquelles la souche L72 est présente ne donnent pas toujours les résultats les plus élevés. On arrive ainsi à un classement des souches de lactobacilles différent lorsqu'elles sont seules ou en associations ; la souche L99 par exemple, est plus protéolytique en association de souches qu'en monosouche.

Les résultats plus faibles observés sur les cultures d'associations de souches pourraient s'expliquer par la technique même d'ensemencement, puisque celui-ci est toujours de $1 \%(\mathrm{v} / \mathrm{v})$ quel que soit le nombre de souches mises en présence. Nous avons vu que la protéolyse des souches de streptocoques mesurée par la technique TNBS était négligeable. Cette faible action protéolytique a déjà été souli- gnée chez les streptocoques lactiques mésophiles (Kikuchi et al, 1973) ; la vitesse à laquelle s'effectue la protéolyse du lait serait insuffisante pour permettre un approvisionnement en acides aminés aux bactéries lactiques et obtenir ainsi un taux de croissance maximal (Juillard et Richard, 1989). Ainsi, dans les associations où la population de lactobacilles est plus faible que dans les cultures de type monosouche, il semblerait normal que la protéolyse soit moins élevée. Toutefois, si on considère que les lactobacilles sont seuls capables de produire une protéolyse visible, les mélanges composés de 3 souches, dont 2 de streptocoques, devraient présenter des valeurs encore plus faibles ; or les mélanges L99 S69 S48 ou L72 S69 S48 par exemple, atteignent respectivement des mesures TNBS similaires à celles des mélanges L99 S69 ou L72 S69. II semble donc que le type d'association mis en jeu doit être pris en compte. II existe vraisemblablement des compétitions plus ou moins importantes pour les acides aminés libres et les petits peptides selon le type de souches mis en présence, comme Juillard et Richard (1989) le soulignent dans l'étude de l'interaction entre souches protéolytiques de streptocoques lactiques mésophiles et leurs variants non protéolytiques.

Par ailleurs, nous avons vu que les associations dans lesquelles la souche S48 est présente sont celles quí donnent les mesures TNBS les plus fortes par rapport aux mélanges comportant la souche S69. Deux hypothèses peuvent être avancées pour expliquer cette différence. Nous pouvons observer que la souche $\$ 48$ acidifie peu le lait au bout de $6 \mathrm{~h}$ de culture par rapport à la souche $S 69$, sans doute parce que cette dernière, utilisant le galactose, a un taux de croissance plus rapide (fig 2); ainsi, l'évolution plus lente de la souche S48 se traduisant par une consommation de substances azotées facilement assimi- 


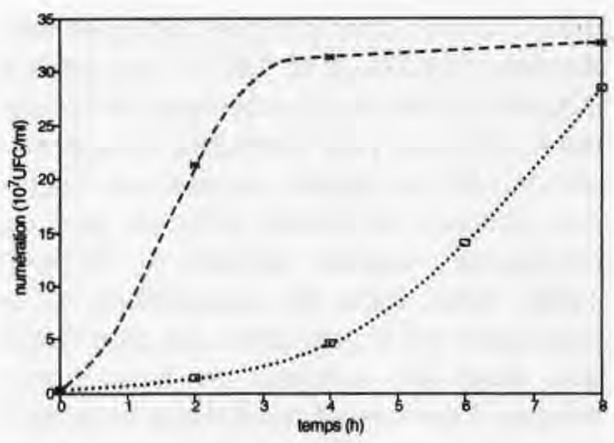

Fig 2. Evolution sur lait des souches de Streptococcus salivarius subsp thermophilus $\mathbf{S 6 9}$ (一*) et S48 (*ロ•*).

Evolution on milk of Streptococcus salivarius subsp thermophilus strains S69 (- - ) and S48 (•ロ•.).

lables, plus faible, permet aux souches de lactobacilles d'atteindre un taux de croissance maximal. II est possible aussi que la présence des 2 types de souches se traduise par un effet synergique notable sur l'activité protéolytique, provoquant une élévation des mesures TNBS, au même titre qu'il existe un effet de synergie sur l'activité acidifiante avec les souches de $S$ salivarius subsp thermophilus et $L$ delbrueckii subsp bulgaricus (Zourari et al; 1992). Une étude plus détaillée des phénomènes d'interaction entre souches devrait permettre d'avancer sur ce problème très complexe, qui n'est pas sans importance en technologie fromagère.

\section{Comparaison de la protéolyse observée sur les cultures et sur les minifromages}

Contrairement aux tests de laboratoire, le niveau moyen des résultats obtenus en associations de souches est en général du même ordre de grandeur que celui observé en monosouches. Signalons que les valeurs de $\mathrm{pH}$ et d'extrait sec des diffé- rentes fabrications en associations de souches sont restées stables et comparables. En revanche, les fromages type monosouche, fabriqués avec la souche de $L$ delbrueckii subsp lactis L85, présentaient un $\mathrm{pH}$ plus faible que les autres fromages.

L'analyse de variance des mesures d'activité protéolytique globale (TNBS), montre qu'il n'y a pas de différence significative au seuil de $5 \%$ entre fromages, qu'ils soient fabriqués avec les monosouches ou avec les associations de souches. Nous pouvons observer dans le cas des monosouches, des écarts-types entre essais différents d'une souche à l'autre, les 2 essais effectués avec la souche L72 sont hétérogènes et rendent la comparaison difficile. Des numérations réalisées sur lait et levains au cours de cette étude n'ont pas montré de réelles différences, permettant d'expliquer cette variation; la souche $L 72$ semble donc être plus sensible à la composition physicochimique de la matière première. Lorsqu'on regarde la hiérarchie des souches obtenue pour chacun des 2 essais, il est intéressant de noter que la souche L99 est toujours la plus protéolytique et la souche $\mathrm{L} 85$ la plus faible. Ce résultat obtenu avec la souche L99 ne concorde pas avec celui obtenu en test de laboratoire qui la plaçait en dernière position; par contre, la souche L85, classée également dans le groupe c (faiblement protéolytique), donne bien sur fromage une protéolyse faible; protéolyse qui peut également s'expliquer par des valeurs de $\mathrm{pH}$ plus faibles (Youssef, 1992). Avec les associations de souches, les résultats des dosages, bien que non significatifs, montrent néanmoins que la souche L99 associée au streptocoque $\mathrm{S} 48$ ou aux 2 streptocoques à la fois (S48 et S69) se retrouve au premier rang, comme dans le cas des fromages type monosouche. Avec le streptocoque S69, c'est la souche L72 qui présente des valeurs plus élevées. 
L'analyse de variance des mesures d'activité aminopeptidasique (Lap) réalisées sur monosouches conduit au même classement que celui obtenu avec les tests de laboratoire; le groupe a est constitué des souches L72, L80 et L99 et le groupe b de la souche L85. En association de souches, la hiérarchie obtenue est différente de celle observée en test de laboratoire. En effet, associée au streptocoque S48, la souche L99 est classée dans le groupe a, au côté des souches $L 72$ et L80, alors qu'en test de laboratoire, elle formait à elle seule le groupe a donnant les valeurs d'activité aminopeptidasique les plus élevées; avec le streptocoque $\$ 69$ seul ou en association avec $S 48$ et $S 69$, la souche L99 est classée dans le groupe b, alors qu'en test de laboratoire elle était classée dans le groupe a; seule la souche L85 présente toujours une faible activité.

L'ensemble de ces observations indique qu'il est difficile de prévoir, à partir des tests de laboratoire, l'intensité de la protéolyse au niveau du fromage, même si le classement de Newman-Keuls conduit aux mêmes groupes homogènes de monosouches dans le cas de l'activité aminopeptidasique. En effet, en association de souches les résultats étant discordants, il est possible que les souches de lactobacilles associées aux streptocoques développent sur lait et sur caillé des activités Lap différentes de celles observées en laboratoire. Desmazeaud et Vassal (1979) ont montré que la capacité de synthèse des dipeptidases de la souche $S$ lactis 261 (Lc lactis subsp lactis) était meilleure lorsque cette souche était cultivée sur lait ou caillé plutôt que sur milieu de laboratoire.

Par ailleurs, si le substrat lait est différent, les conditions de température choisies peuvent influencer la lyse cellulaire et donc la protéolyse. On sait que si les bactéries lactiques, qui possèdent des systèmes protéolytiques extracellulaires ou liés aux enveloppes, sont capables d'hy- drolyser les caséines et les peptides en phase exponentielle de croissance, elles possédent également des systèmes protéolytiques intracellulaires qui sont libérés en phase de déclin lorsque les cellules se lysent. L'importance de la lyse des cellules des levains dans le fromage jeune influence donc la production d'acides aminés; or cette lyse, si elle est dépendante des propriétés autolytiques des levains, est aussi dépendante des conditions du milieu et d'affinage, en particulier du couple temps-température, du niveau d'acide lactique et du taux de diffusion du sel dans le fromage (Youssef, 1992).

D'autre part, pour être active, une enzyme doit être stable dans le fromage. Peu de travaux ont été réalisés sur les facteurs déterminant la stabilité des enzymes protéolytiques des levains dans les conditions d'affinage. Cependant, Law et al (1974) ont montré que des préparations de dipeptidases de LC lactis subsp cremoris NCDO924 pouvaient être suffisamment stables pour persister dans le Cheddar pendant au moins 3 mois; de même, EI Abboudi et al (1992) ont mis en évidence la stabilité, pendant au moins 2 mois, d'une aminopeptidase dans le Cheddar.

Enfin, si le potentiel protéolytique des levains contribue de façon non négligeable à la protéolyse du fromage, les enzymes naturelles du lait (Humbert et Alais, 1979) peuvent également jouer un rôle dans la dégradation des protéines et expliquer les discordances observées sur cultures de souches et sur fromages.

L'ensemble de ces résultats montre qu'en utilisant des tests simples de mesure de l'activité protéolytique de souches (TNBS et Lap), on obtient des informations contradictoires, selon que ces tests sont appliqués en laboratoire sur milieu lait ou synthétique ou sur fromages. Les tests de laboratoire n'ont comme intérêt que le classement des monosouches; en revanche, 
les minifabrications devraient permettre une meilleure approche du potentiel protéolytique des souches. Les minifabrications type monosouche s'éloignant de la technologie Comté où les levains sont toujours composés de 2 types de souches, streptocoques et lactobacilles, il n'apparaît pas nécessaire de retenir ce test comme critère de classement des souches. Si les minifabrications de type associations de souches utilisées au cours de ce travail apportent des informations utiles, cette caractérisation des souches dans des conditions standard reste insuffisante. En effet, la matière première, la technologie de fabrication ainsi que l'affinage agissent de manière significative sur la protéolyse du fromage. A partir de fromage modèle développé par Noomen (1978), Youssef (1992) a montré que la production d'acides aminés était améliorée lorsque le $\mathrm{pH}$ du fromage est élevé, le taux de sel bas et la température d'affinage haute. II est vraisemblable que le potentiel protéolytique des souches s'exprime de manière différente selon les conditions choisies; cet aspect devrait être pris en compte pour la caractérisation des souches. II reste également à démontrer que les 2 tests qui ont été choisis (TNBS et Lap), même lorsqu'ils sont appliqués sur minifromages, sont suffisamment appropriés et discriminants pour que les résultats obtenus soient en relation étroite avec la nature et l'intensité de la protéolyse observée dans les fromages.

\section{REMERCIEMENTS}

Nous remercions JC Gripon et D Lebars de la station de recherches laitières de I'INRA de Jouy-en-Josas, pour leurs conseils sur les techniques de mesures de l'activité protéolytique des bactéries lactiques, A Berodier pour la mise au point du dosage de l'activité leucineaminopeptidase sur fromage, et G Duboz pour la réalisation des fabrications fromagères.
Ce programme a reçu le soutien financier du Comité interprofessionnel du gruyère de Comté, de l'Association pour le développement de la recherche dans l'industrie laitière et de l'Association nationale pour la recherche et la technologie (bourse CIFRE de $Y$ Bouton, convention n०12/89).

\section{RÉFÉRENCES}

Anonyme (1985) Lait. Définition et évaluation de la précision globale des méthodes indirectes d'analyse du lait. Application au calibrage et au contröle de qualité. Norme FIL provisoire 128 , Bruxelles

Bouillanne C, Desmazeaud MJ (1980) Ėtude de quelques caractères de souches de Streptococcus thermophilus utilisées en fabrication de yoghourt et proposition d'une méthode de classement. Lait 60, 458-473

Bouton Y (1992) Caractérisation approfondie sur minifromages de l'activité protéolytique de souches et associations de souches de bacteries lactiques thermophiles utilisées en fabrication de Comté. Relation avec les caractéristiques organoleptiques. Thèse de doctorat, Nancy I, France

Cardenas R, Duboz G, Grappin R (1991a) Minifromagerie automatisée de I'INRA de Poligny: I'investigation en laboratoire de l'ensemble de la fabrication. Process 1063, 2831

Cardenas R, Duboz G, Grappin R (1991b) Minifromagerie automatisée de I'INRA de Poligny : la fabrication. Process 1065, 66-69

Cholette H, Mc Kellar RC (1990) Influence of pH on the properties of Lactobacillus helveticus aminopeptidase. J Dairy Sci 73, 2278-2286

Desmazeaud MJ (1990) Rôles des cultures de micro-organismes dans la flaveur et la texture des produits laitiers fermentés. $23 e$ Congr Int Lait, Montréal, 1555-1577

Desmazeaud MJ, Vassal L (1979) Activité protéolytique intracellulaire de streptocoques lactiques mésophiles. Rôle au cours de l'affinage des fromages. Lait 59, 327-344

El Abboudi M, El Soda M, Pandian S, Barreau M, Trepanier G, Simard RE (1992) Peptidase activities in debittering and nondebittering strains of Lactobacilli. Int Dairy J 2, 55-94 
El Soda M, Desmazeaud MJ (1982) Les peptide-hydrolases des lactobacilles du groupe Thermobacterium. 1. Mise en évidence de ces activités chez Lactobacillus helveticus, $L$ acidophilus, $L$ lactis et $L$ bulgaricus. Can $J$ Microbiol 28, 1181-1188

Fox PF (1989) Proteolysis during cheese manufacture and ripening. J Dainy Sci 72, 13791400

Gripon JC (1986) Les ferments lactiques thermophiles, étude de leur activité protéolytique. Rapport final, contrat CEE/ITG/INRA N ${ }^{0} 1150 /$ 86, code INRA 9182A

Humbert G, Alais C (1979) Review of the progress of dairy science. The milk proteinase system. J Dairy Sci 46, 559-571

Juillard V, Richard J (1989) Étude de l'interaction entre souches protéolytiques de streptocoques lactiques mésophiles et leurs variants non protéolytiques, au cours de leur croissance dans le lait. Lait 69, 291-304

Khalid NM, Marth EH (1990) Partial purification and characterization of an aminopeptidase from Lactobacillus helveticus CNRZ 32. Syst Appl Microbiol 13, 311-319

Kikuchi T, Desmazeaud M, Bergère JL (1973) Aptitude des streptocoques lactiques à la protéolyse. I. Étude de l'action de streptocoques lactiques mésophiles sur les constituants azotés du lait. Lait 53, 369-385

Kuchroo CN, Fox PF (1982) Soluble nitrogen in Cheddar cheese : comparison of extraction procedures. Milchwissenschaft 37, 331-335

Law BA, Sharpe ME, Reiter B (1974) The release of intra-cellular dipeptidase from starter streptococci during Cheddar cheese ripening. J Dairy Res 41, 137-146

Linden G, Humbert G, Desnouveaux R, Picard J (1982) Applications de la dissolution complète du lait à la détermination de quelques activités enzymatiques. Lait 62, 209-219

Mc Kellar (1981) Development of off-flavors in ultra high temperature and pasteurized milk as a function of proteolysis. J Dairy Sci 64, 2138-2145
Meyer J, Howald D, Jordi R, Fürst M (1989) Location of proteolytic enzymes in Lactobacillus lactis and Streptococcus thermophilus and their influence on cheese ripening. Milchwissenschaft 44, 678-681

Noomen A (1978) Activity of proteolytic enzymes in simulated soft cheeses (Meshanger type) 1. Activity of milk protease. Neth Milk Dairy J 32, 24-48

Oberg CJ, Khayat FA, Richardson GH (1990) Proteinase profiles of Lactococcus lactis subsp cremoris using high performance liquid chromatography. J Dairy Sci 73, 1465-1471

Oberg CJ, Weimer BC, Moyes LV, Brown RJ, Richardson GH (1991) Proteolytic characterization of Lactobacillus delbrueckii ssp bulgaricus strains by the o-phthaldialdehyde test and amino acid analysis. J Dairy Sci 74, 398403

Rabier D, Desmazeaud MJ (1973) Inventaire des différentes activités peptidasiques intracellulaires de Streptococcus thermophilus. Purification et propriétés d'une dipeptide- hydrolase et d'une aminopeptidase. Biochimie 55, 389-404

Rollema HS, Mc Kellar RC, Sorhaug T, Surhen G, Zadow JG, Law BA, Poll JK, Stepaniak L, Vagias $G$ (1989) Comparison of different methods for the detection of bacterial proteolytic enzymes in milk. Milchwissenschaft 44, 491496

Thomas TD, Pritchard GG (1987) Proteolytic enzymes of dairy starter cultures. FEMS Microbiol Rev 46, 245-268

Youssef YB (1992) Factors affecting proteolytic action of Lactococcus lactis in cheese. Ph D thesis, Agricultural University, Wageningen.

Zourari A, Accolas JP, Desmazeaud MJ (1992) Metabolism and biochemical characteristics of yogurt bacteria. A review. Lait 72, 1-34

Zourari A, Roger S, Chabanet C, Desmazeaud MJ (1991) Caractérisation des bactéries lactiques thermophiles isolées de yaourts artisanaux grecs. I. Souches de Streptococcus salivarius subsp thermophilus. Lait 71, 445-461 